\title{
The GERDA Neutrinoless-Double-Beta decay experiment
}

\section{Béla MAJOROVITS* for the GERDA collaboration}

Max-Planck-Institut für Physik

E-mail: bela@mppmu.mpg •de

Neutrinoless double beta $(0 v \beta \beta)$-decay is a key process to gain understanding of the nature of neutrinos. The GErmanium Detector Array (GERDA) is designed to search for $0 v \beta \beta$-decay of the isotope ${ }^{76} \mathrm{Ge}$. Germanium crystals enriched in ${ }^{76} \mathrm{Ge}$, acting as source and detector simultaneously, will be submerged directly into an ultra pure cooling medium that also serves as a radiation shield. This concept will allow for a reduction of the background by up to two orders of magnitudes with respect to earlier experiments. The experiment is currently being installed in hall A of the underground laboratory of the LNGS, INFN in Italy. Data taking is expected to start in 2009.

PACS: $23.40 .-\mathrm{s}, 12.15 . \mathrm{Ff}, 14.60 . \mathrm{St}, 29.40 . \mathrm{Wk}$

Identification of dark matter 2008

August 18-22, 2008

Stockholm, Sweden

${ }^{*}$ Speaker. 

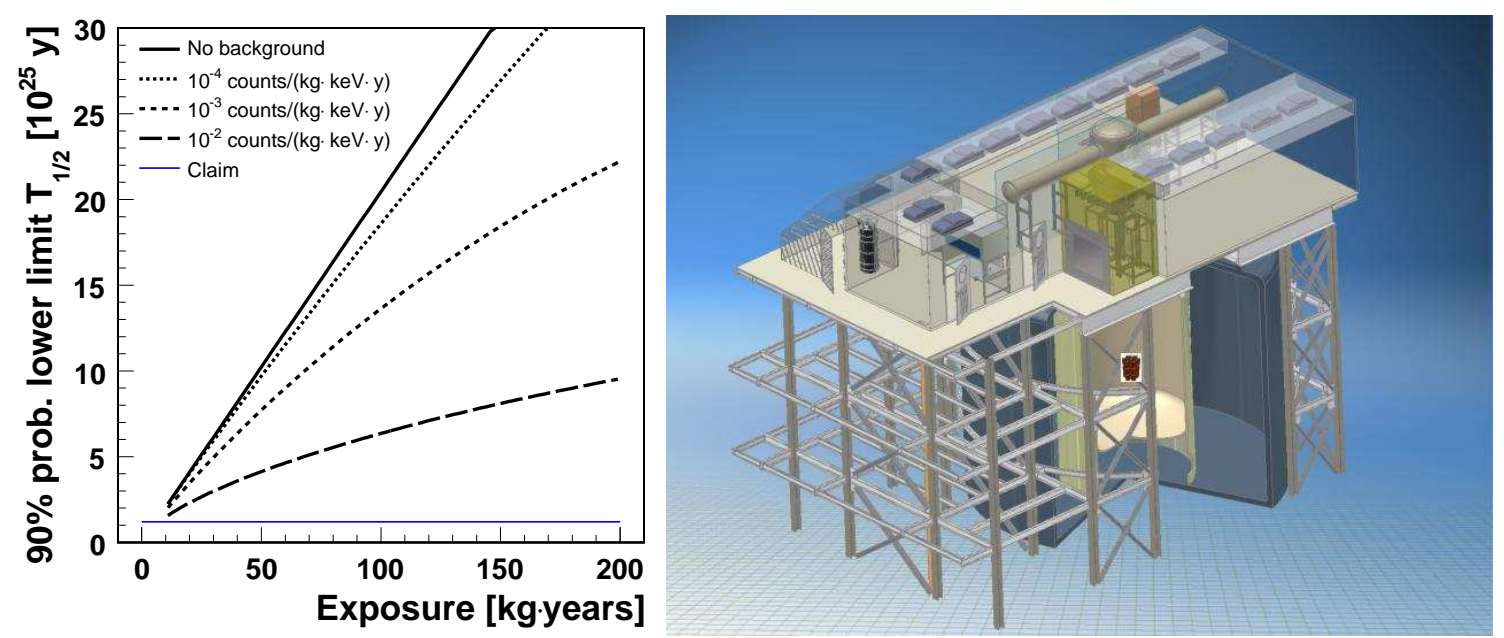

Figure 1: Left: Expected 90\% probability lower limit on $\mathrm{T}_{1 / 2}^{0 v \beta \beta}$ as a function of exposure for different background indices (taken from [6]). Right: Schematic view of the GERDA setup. The detector array is sitting in the center of the cryostat. The cryo tank is surrounded by a water buffer serving as additional shield and as a muon-Čerenkov veto system. The detector array is loaded through a lock system located in a clean-room on the top of the tank.

The GERmanium Detector Array, GERDA [1], is designed to search for the neutrinoless double beta-decay $(0 v \beta \beta)$ of ${ }^{76} \mathrm{Ge}$. Neutrino accompanied double beta-decay is an allowed second order weak process. Only if the neutrino is a massive Majorana-particle the decay can also occur without the emission of a neutrino [2]. Observation of the $0 v \beta \beta$-decay would have far reaching consequences for neutrino physics. The importance of such a search is emphasized by the observation of a non-zero neutrino mass of yet unknown scale from flavor oscillations [3]. The observation of neutrino-oscillation do allow for two different mass scenarios with a normal or an inverted hierarchy [4]. These can be disentangled by $0 v \beta \beta$-decay for a Majorana neutrino if a sensitivity for the effective Majorana neutrino-mass of $\approx 10 \mathrm{meV}$ can be achieved [5].

The most stringent $0 v \beta \beta$ half-life limits so far are based on High-Purity-Germanium, HPGe, detector technology. The background level, the energy resolution, the detection efficiency and the enrichment in the isotope that decays through double beta-decay determine the sensitivity of an $0 v \beta \beta$-decay experiment. HPGe detectors have the advantage that they serve as source and detector simultaneously, that they are intrinsically very clean and that they have a very good energy resolution. As the natural abundance of ${ }^{76} \mathrm{Ge}$ is only $7.4 \%$ the materials used for detector production is enriched to up to $88 \%$ in ${ }^{76} \mathrm{Ge}$. Simultaneously it is mandatory to minimize the background.

Currently the Heidelberg-Moscow (HdM) and IGEX experiments give limits on the lifetime of $0 v \beta \beta$-decay of $1.9 \cdot 10^{25} \mathrm{y}$ and $1.6 \cdot 10^{25} \mathrm{y}$, respectively (90\% C.L.) $[7,8]$. These lower limits can be translated into upper limits for the effective Majorana neutrino-mass in the range between $0.35 \mathrm{eV}$ $1.2 \mathrm{eV}$ and $0.3 \mathrm{eV}-1.5 \mathrm{eV}$ for the two experiments for different matrix elements (see $[7,8]$ and references therein). A part of the HdM collaboration claims to have observed a peak at $\mathrm{Q}_{\beta \beta}$ with $4.2 \sigma$ confidence level which can be attributed to $0 v \beta \beta$-decay with a half-life of $\mathrm{T}_{1 / 2}^{0 v \beta \beta}=1.19_{-0.50}^{+2.99}$. $10^{25}$ y (3 $\sigma$ range) [9].

GERDA is currently being installed in Hall A of the Gran Sasso underground laboratory 

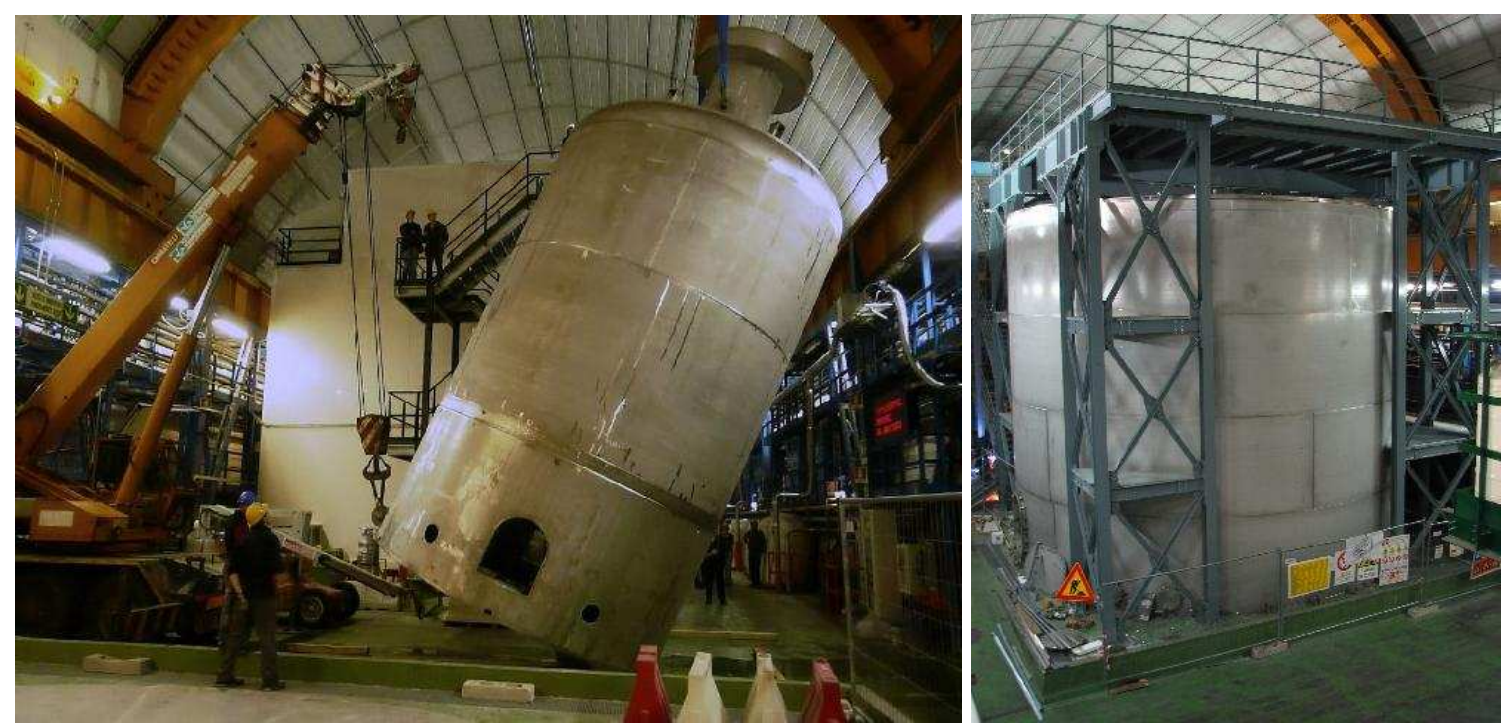

Figure 2: Left: Arrival of the cryostat at hall A in LNGS. Right: GERDA tank and building as of Oct. 2008.

(LNGS), Italy. The conceptual design bases on the idea of using an ultra-pure cryogenic liquid as the cooling medium and as a shield against gamma radiation simultaneously [10]. A cryostat (see Fig 2 left) made out of carefully selected stainless steel will house an array of HPGe detectors. The cryostat has a low-background copper inlet as a radiation shield against the steel of the cryotank. The cryogenic volume is surrounded by a buffer of ultra-pure water acting as an additional gamma and neutron shield. The water buffer is additionally used as a muon-Čerenkov veto [1]. The setup is schematically depicted in Fig. 1.

The experiment is foreseen to proceed in two phases. In the first phase, enriched detectors which were previously operated by the HdM and IGEX collaborations will be re-deployed. If the HdM claim is correct, in the region of interest $(6.0 \pm 1.4)$ events above a background of 0.5 events after one year of measurement $(15 \mathrm{~kg} \cdot \mathrm{y})$ are expected. This will be enough to either confirm or refute the claim from the HdM experiment (see Fig. 1). In the second phase custom made segmented detectors will be installed. An exposure of $100 \mathrm{~kg} \cdot \mathrm{y}$ with a background level of $10^{-3}$ counts $/(\mathrm{keV} \cdot \mathrm{kg} \cdot \mathrm{y})$ is foreseen. A lower limit on the half-life of $0 v \beta \beta$-decay of higher than $1.5 \cdot 10^{26}$ y corresponding to an upper limit of $\approx 140 \mathrm{meV}$ (using the matrix elements from [11]) can be set with $90 \%$ probability if no signal is observed.

All IGEX and HdM as well as the detectors previously used for the Genius-TF [12] are presently stored underground at the LNGS in Italy. The IGEX and HdM detectors have been removed from their conventional cryostats and reprocessed without any technical problems. A prototype detector has been successfully operated in liquid argon in the low background copper holder at LNGS for several months. Energy resolution and leakage current measurements have been performed with some of the reprocessed enriched detectors in a test bench in the LNGS underground laboratory. The energy resolution (FWHM) at $1.3 \mathrm{MeV}$ was less then $4 \mathrm{keV}$ with similar electronics as will be used in the GERDA setup for all detectors.

The material for phase II of the experiment, $35.5 \mathrm{~kg}$ of germanium enriched to $87 \%-88 \%$ in ${ }^{76} \mathrm{Ge}$ in form of $\mathrm{GeO}_{2}$ is presently stored underground. The yields and efficiencies of the reduction 

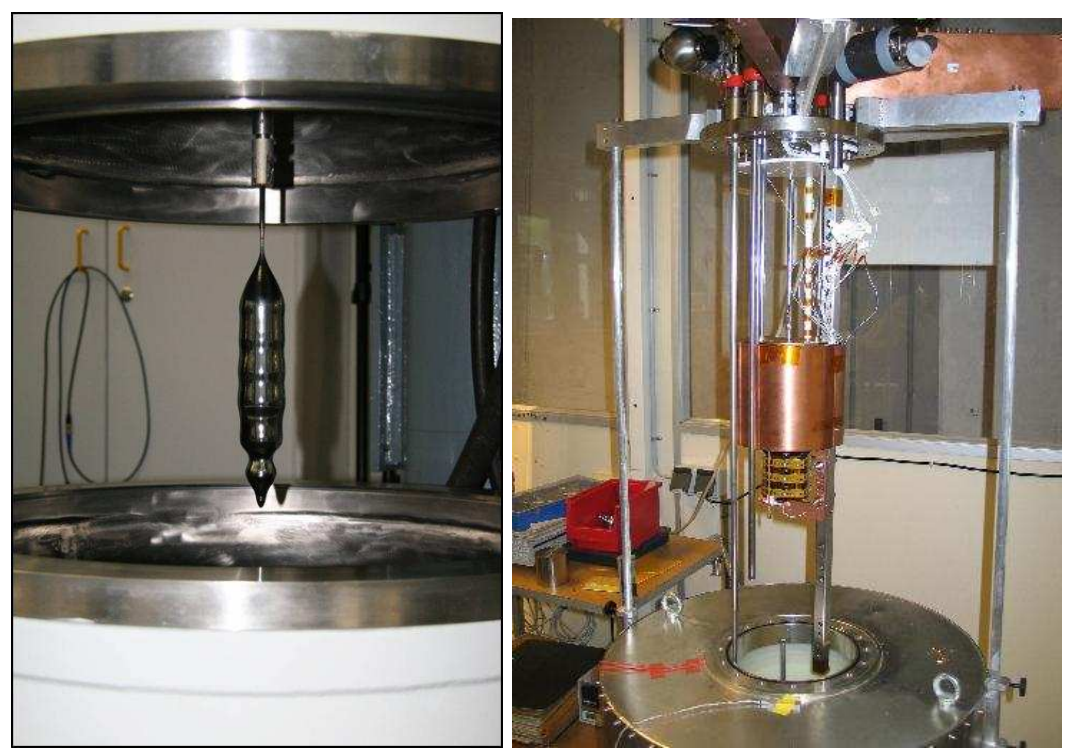

Figure 3: Left:Germanium crystal pulled with the dedicated Czochralski puller at IKZ, Berlin. Right: 18fold segmented HPGe detector in the test stand. The detector was operated directly in liquid nitrogen.

and purification processes have been studied with isotopically shifted Ge material. It was shown that $6 \mathrm{~N}$ material can be reliably produced with a yield of above $80 \%$ without isotopic dilution. Processing of the enriched material is scheduled for the near future. R \& D towards the pulling of HPGe crystals is ongoing at the Institut für Kristallzüchtung in Berlin. First Ge crystals have been pulled in a dedicated Czochralski puller (see Fig. 3). The infrastructure for characterization of their impurity levels using Photo-thermal Ionization Spectroscopy (PTIS) [13] is in place.

The nominal phase II design foresees 18 -fold segmented true coaxial n-type detectors. The segmentation helps to identify multiple Compton-scattering events in the region of interest. As shown in [14] the Compton background can be identified with high efficiency depending on its source and location. For the first time ever a segmented n-type HPGe detector was operated successfully in a cryo-liquid. The detector was running for more than four months without deterioration of the performance. A core resolution of $4 \mathrm{keV}$ and segment resolutions between $4 \mathrm{keV}$ and $7 \mathrm{keV}$ at the $1.3 \mathrm{MeV}$ calibration peak were measured (see Fig. 4 for calibration spectra). The leakage current of the detector was monitored on a regular basis. It remained below $6 \mathrm{pA}$ for the whole measurement period.

As a conclusion it can be stated that the installation of the GERDA experiment is well on its way. The infrastructure at LNGS in Italy is close to being finished while detectors for phase I of the experiment have been prepared. It has been shown that the nominal phase II technique of operating 18-fold segmented n-type HPGe detectors with the novel low mass contacting technique is suitable for a cryogenic liquid surrounding.

\section{References}

[1] GERDA proposal to the LNGS P38/04, I. Abt et al., hep-exp/0404039 

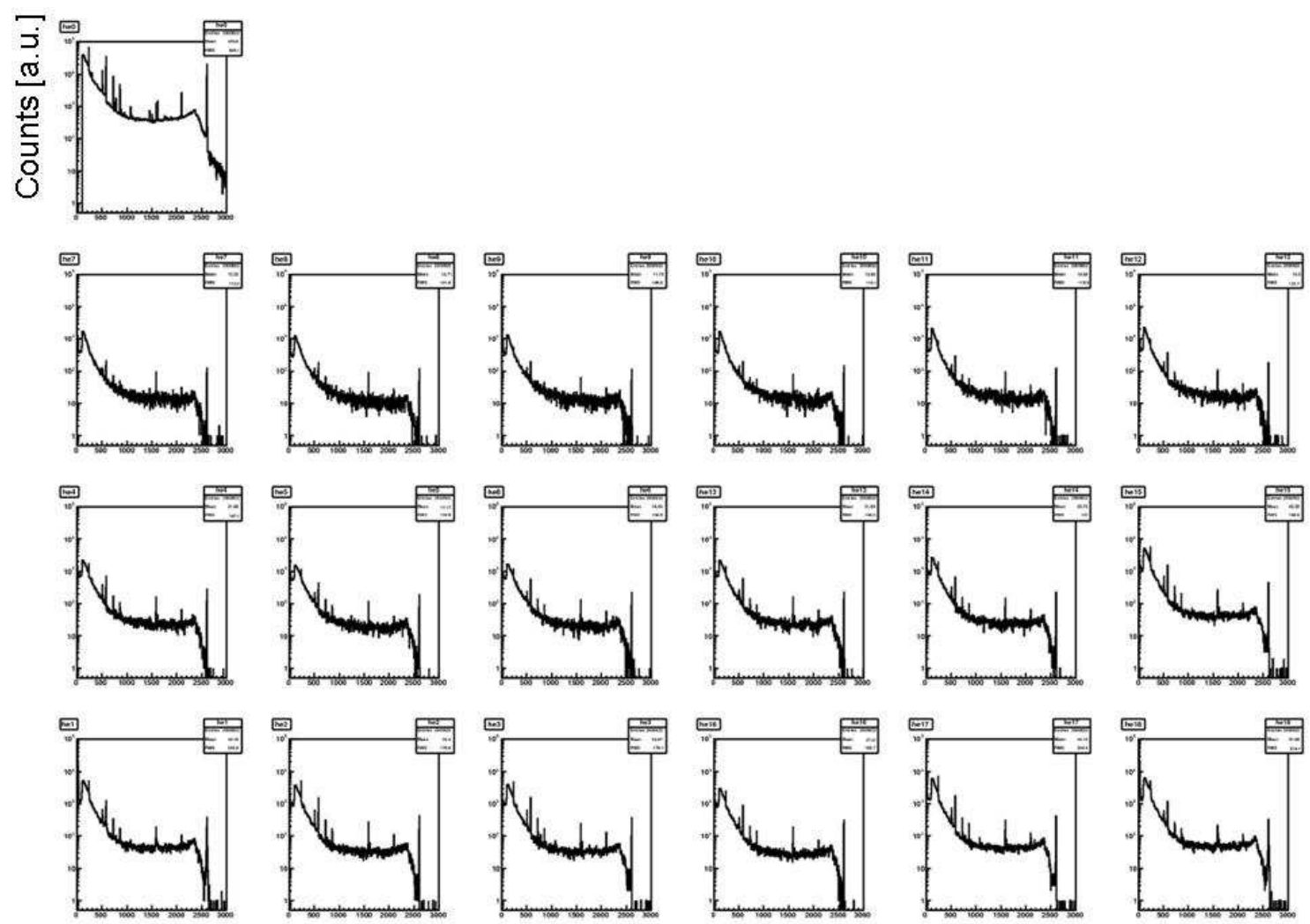

Energy $[\mathrm{keV}]$

Figure 4: Calibration spectra of the core and all 18 segments of the segmented n-type HPGe detector operated in liquid nitrogen taken with a ${ }^{228} \mathrm{Th}$ source.

[2] S. Elliott and P. Vogel, Ann. Rev. Nucl. Part. Sci 52(2002)115

[3] S. Fakuda et al., Phys. Rev. Lett. 82(1999)2644 and C. Ahmad et al., Phys. Rev. Lett 92(2004)181301

[4] S. Bilenky et al., Phys. Rev. C 64(2001)053010

[5] F. Feruglio et al., Nucl. Phys. B 637(2002)345

[6] A. Caldwell and K. Kröninger, Phys. Rev. D 74(2006)092003

[7] L. Baudis et al., Phys. Rev. Lett. 83(1999)41

[8] C. Aalseth et al., Phys. Rev. D 65(2002)092007

[9] H.V. Klapdor-Kleingrothaus et al., Phys. Lett. B 586(2004)198

[10] G. Heusser, Ann. Rev. Nucl. Part. Sci. 45 (1995)543

[11] V. Rodin et al., Nucl. Phys. A 766(2006)107 and V. Rodin, et al., arXiv:0704.4304

[12] H.V. Klapdor-Kleingrothaus, Nucl.Instrum.Meth. A 481(2002)149

[13] E. Haller, W. Hansen and F. Goulding, IEEE Trans. Nucl. Sci, 22(1975)127

[14] I. Abt et al., Nucl. Instr. Meth. A 570(2007)479, I. Abt et al., Nucl. Instr. Meth. A 583(2007)332 and I. Abt et al., Eur. Phys. J. C 52(2007)19 